\title{
† CEREUS
} Atendimento fisioterapêutico em Clínica Escola de uma Faculdade privada no
interior do Tocantins - Brasil: Satisfação dos usuários

Physiotherapeutic care in a school clinic of a private faculty of the interior of Tocantins -

Brazil: user satisfaction

lara Sousa Aragão, Karla Camila Correia da Silva², Rogério Carvalho

Figueredo 3

As clínicas escolas das faculdades podem ser consideradas diferenciais na formação dos profissionais, por oportunizarem a realização dos estágios supervisionados, onde os acadêmicos podem desenvolver suas habilidades e competências junto aos pacientes, tornando importante verificar a satisfação dos usuários das clínicas quanto aos atendimentos realizados pelos acadêmicos. Este estudo objetivou avaliar o nível de satisfação dos pacientes que realizam atendimentos na Clínica Escola de Fisioterapia do Instituto Educacional Santa Catarina - Faculdade Guaraí (IESC-FAG). Trata-se de uma pesquisa de campo descritiva de abordagem quanti-qualitativa. Os pacientes participaram da pesquisa por meio de um questionário contendo 27 perguntas objetivas, relacionadas à Clínica Escola e aos serviços prestados. Foram entrevistados 72 voluntários, no período entre junho a outubro de 2018, prevalecendo o sexo feminino $(80 \%)$, com renda variável entre 1 e 3 salários mínimos (95\%), baixo nível de escolaridade e que foram indicados através de amigos. Relataram $100 \%$ de satisfação geral com os atendimentos e satisfação das condições, e afirmaram que indicam a clínica para familiares e amigos, e retornariam para novos atendimentos. Apontaram como itens que precisam de melhorias: estacionamento, maior privacidade nos atendimentos e melhora da recepção. Os voluntários mostraram-se satisfeitos com os serviços, sugerindo algumas mudanças para seu aprimoramento.

Palavras-chave: Avaliação em saúde. Serviços de saúde. Satisfação do paciente. Fisioterapia.

\section{ABSTRACT}

School clinics at colleges in the health field can be considered differentials in the training of future professionals, as they make it possible to carry out supervised internships, where academics can develop their skills and competences with patients. To this end, it is important to verify the satisfaction of users of the clinics regarding the care provided by academics. This study aimed to assess the level of satisfaction of patients who attend at the Clínica Escola de Fisioterapia of Instituto Educacional Santa Catarina Faculdade Guaraí (IESC-FAG). This is a descriptive field research with a quantitative and qualitative approach. Patients participated in the research through a questionnaire containing 27 objective questions, related to Clínica Escola and the services provided by academics. 72 volunteers were interviewed, between June and October 2018, with a predominance of females $(80 \%)$, with variable income between 1 and 3 minimum wages (95\%), low level of education and who were referred to the school clinic through of friends and satisfaction of the conditions of the Clínica Escola. Everyone is satisfied with the therapeutic services offered and stated that they recommend the clinic to family and friends, and would return for new services. They pointed out as items that need improvement: parking, greater privacy in attendance and improved reception. The volunteers were satisfied with the services, suggesting some changes for their improvement.

Keywords: Health assessment. Health services. Patient satisfaction. Physiotherapy.
Fisioterapeuta. Educacional Santa Faculdade Guaraí.

E-mail:

iarasousa13@hotmail.com

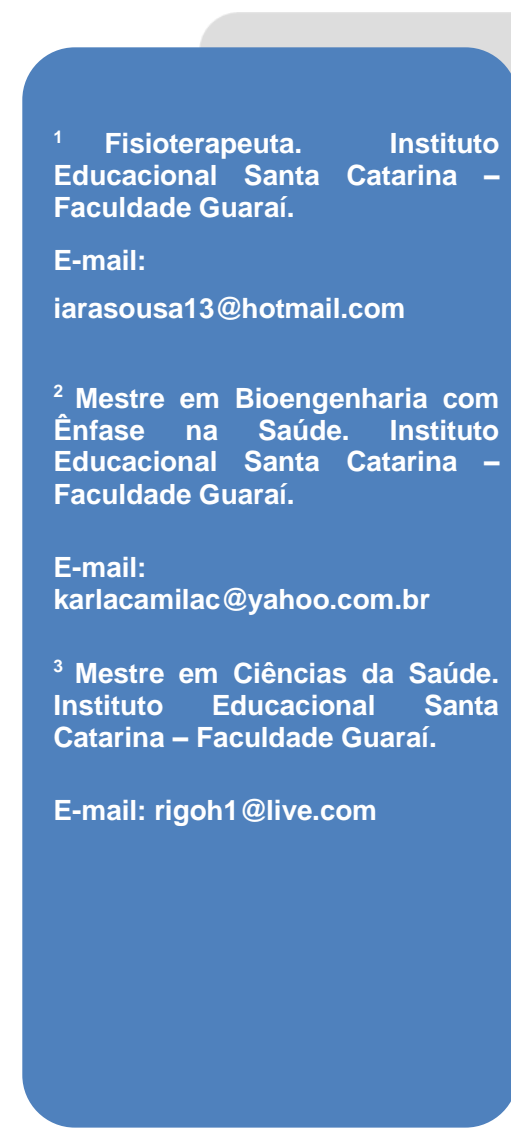




\section{INTRODUÇAOO}

Os estágios supervisionados para os cursos na área da saúde constituem-se em atividade curricular de ordem prática permitindo que o estagiário aplique a teoria à realidade profissional, mediante trabalho supervisionado nas mais variadas áreas de atuação, devendo o estagiário seguir, cumprir e fazer cumprir o código de ética profissional na sua totalidade, em conformidade com as principais leis e diretrizes da educação (GAIAD, PEIXOTO, GONÇALVES, 2005).

No curso de bacharel em fisioterapia do Instituto Educacional Santa Catarina Faculdade Guaraí (IESC/FAG), os estágios supervisionados são realizados do sétimo ao décimo períodos de acordo com a matriz curricular vigente a partir do semestre de 2015 , sendo $20 \%$ da carga horária total do curso, que deve ser cumprida obrigatoriamente na íntegra (BRASIL, 2002).

A Clínica Escola de Fisioterapia (CEF) faz parte da Clínica de Educação para a Saúde (CEPS) do Instituto Educacional Santa Catarina - Faculdade Guaraí. Ela foi inaugurada em maio de 2012, tendo como objetivo integrar o ensino e a assistência à saúde da população de Guaraí e cidades circunvizinhas. Em parceria com a prefeitura municipal, oferece a comunidade atendimentos nas áreas de fisioterapia, enfermagem e medicina.

A CEF promove aos acadêmicos do curso de fisioterapia práticas de estágios curriculares junto à comunidade local, complementando a formação acadêmica dos futuros profissionais. Ela foi inaugurada em agosto de 2016 e está em funcionamento há três anos realizando atendimentos nas áreas de fisioterapia, de forma gratuita à população de Guaraí e região. São realizados atendimentos nas áreas de: neurofuncional, traumato-ortopedia, cardiorrespiratória, uroginecologia, pediatria, dermato-funcional e geriatria. O quantitativo geral de atendimentos realizados dentro da clínica escola, no primeiro semestre de 2019 , foi de 2.618 atendimentos prestados.

Diante disso, o contentamento do paciente é importante na medida de qualidade dos serviços, pois proporciona informações sobre os resultados do mantenedor dos atendimentos em relação aos valores e expectativas do cliente, além de constituir um instrumento valioso de abordagem, administração e planejamento (JESUS et al. 2010).

De acordo com Brito, Jesus e Fernandes (2012) estas práticas de avaliação da satisfação exigem a realização de avaliações, interferências e reflexões em processos fisioterapêuticos, apontando o avanço do conhecimento científico, a formação de 
discentes e o atendimento às necessidades da população na recuperação funcional e na promoção da qualidade de vida.

Essas pesquisas envolvendo clínicas escolas vêm sendo cada vez mais realizadas, sendo direcionando trabalhos sobre a área social, procurando verificar a expectativa da satisfação dos pacientes em relação ao atendimento prestado nesses serviços. Nesse contexto, questionou-se: "Qual o nível de satisfação dos pacientes atendidos na Clínica Escola de Fisioterapia da IESC-FAG?"

A fisioterapia apresenta uma série de características que influenciam a satisfação do cliente: a interação com o paciente sempre leva mais tempo do que uma consulta médica por exemplo, pois a terapia envolve maior contato físico, exigindo a participação ativa do paciente.

Portanto, o objetivo geral desse trabalho foi verificar a satisfação dos pacientes atendidos na CEF do IESC-FAG, e os objetivos específicos foram: avaliar a satisfação dos pacientes em relação aos recursos físicos da Clínica de Escola de Fisioterapia; avaliar a satisfação dos pacientes em relação ao relacionamento interpessoal da Clínica de Escola de Fisioterapia; verificar como o paciente teve o conhecimento sobre os atendimentos da Clínica Escola de Fisioterapia.

\section{MATERIAIS E METODOS}

Tratou-se de uma pesquisa de campo, descritivo e de abordagem quantiqualitativa. Este estudo foi aprovado pelo Comitê de Ética em Pesquisa (CEP) da Universidade Estadual do Tocantins (UNITINS), com o parecer de número 2.930.785 CAAE 89902217.3.0000.8023. Para coleta de dados utilizou-se um questionário aplicado durante os meses de agosto a dezembro de 2018.

Foram entrevistados 72 (setenta e dois) pacientes atendidos nas áreas de traumato-ortopedia, cardiorrespiratória, dermato-funcional, neurofuncional, uroginecologia e geriatria.

Os pacientes foram abordados na recepção da CEF, antes ou após o atendimento fisioterapêutico, onde foi aplicado o TCLE, e um questionário contendo 27 perguntas, dividido em tópicos: dados sociodemográficos e clínicos; aspectos interpessoais na interação usuário-terapeuta; ambiente físico; acesso ao serviço e atendimento do recepcionista e pessoal de apoio. A pesquisa foi realizada somente com os pacientes maiores de 18 anos de idade. Os dados coletados foram tratados com estatística 
descritiva apresentados em tabelas com percentis. $E$ analisados qualitativamente a partir de discussão teórica com a literatura atual relacionada ao tema.

\section{RESULTADOS}

A pesquisa foi realizada no CEPS do IESC/FAG, onde fica localizada a CEF, situada Rua Juscelino Kubitschek, número 2529-2605, Setor Universitário, Guaraí - TO, Brasil.

Figura 1: Entrada do CEPS, onde fica situada a CEF da IESC-FAG.

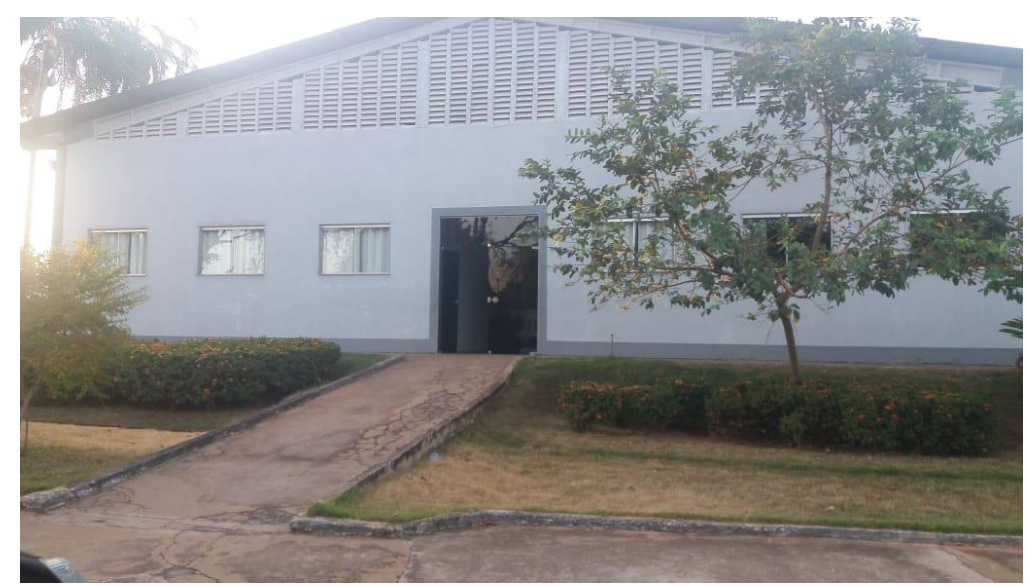

Fonte: Arquivo pessoal dos autores.

A amostra de 72 (setenta e dois) participantes foi distribuída conforme as especialidades de serviços oferecidos, conforme descrito na tabela 1 a seguir.

Tabela 1. Quantitativo de participantes de acordo com as especialidades dos serviços oferecidos na CEF do IESC-FAG.

\begin{tabular}{cc}
\hline Especialidades dos serviços & Quantidade de participantes (N) \\
\hline Ortopedia & 20 \\
\hline Neurofuncional & 12 \\
\hline Dermato-funcional & 14 \\
\hline Cardiologia & 4 \\
\hline Geriatria & 12 \\
\hline Uroginecologia & 10 \\
\hline Total & 72 \\
\hline
\end{tabular}

Fonte: Elaborado pelos autores. 
Percebe-se que as especialidades com maior número de participantes foram de Ortopedia e Dermato-funcional, podendo caracterizar assim, as áreas com maior demanda de atendimentos na CEF.

Os critérios de avaliação da satisfação dos usuários foram verificados de acordo com cada especialidade, sendo identificadas conforme descrição: Ortopedia (Orto); Neurofuncional (Neuro); Dermato-funcional (Dermato); Cardiologia (Cardio); Geriatria (Geri) e Uroginecologia (Urogi).

As características sociodemográficas dos participantes da pesquisa estão apresentadas na tabela 2 a seguir.

Tabela 2. Dados Sociodemográficos dos pacientes da CEF do IESC-FAG.

\begin{tabular}{|c|c|c|c|c|c|c|}
\hline VARIÁVEIS & Orto & Neuro & Dermato & Cardio & Geri & Urogi \\
\hline \multicolumn{7}{|l|}{ SEXO } \\
\hline Masculino & $20 \%$ & $42 \%$ & - & $75 \%$ & $50 \%$ & - \\
\hline Feminino & $80 \%$ & $58 \%$ & $100 \%$ & $25 \%$ & $50 \%$ & $100 \%$ \\
\hline \multicolumn{7}{|l|}{ ESCOLARIDADE } \\
\hline $1^{\circ}$ grau incompleto & $35 \%$ & $8 \%$ & $14 \%$ & $50 \%$ & $50 \%$ & $30 \%$ \\
\hline $1^{\circ}$ grau completo & - & $17 \%$ & $7 \%$ & $25 \%$ & - & $20 \%$ \\
\hline $2^{\circ}$ grau incompleto & $5 \%$ & - & - & - & - & - \\
\hline $2^{\circ}$ grau completo & $35 \%$ & $75 \%$ & $36 \%$ & $25 \%$ & $50 \%$ & $20 \%$ \\
\hline $3^{\circ}$ grau incompleto & - & - & $36 \%$ & - & - & $30 \%$ \\
\hline $3^{\circ}$ grau completo & $25 \%$ & - & $7 \%$ & - & - & - \\
\hline \multicolumn{7}{|l|}{ RENDA FAMILIAR } \\
\hline Abaixo de um salário & - & - & - & - & - & - \\
\hline 1 a 3 salários & $95 \%$ & $83 \%$ & $50 \%$ & $75 \%$ & $50 \%$ & $80 \%$ \\
\hline 4 a 6 salários & $5 \%$ & $17 \%$ & $43 \%$ & $25 \%$ & $50 \%$ & $20 \%$ \\
\hline Acima de 6 salários & - & - & $7 \%$ & - & - & - \\
\hline \multicolumn{7}{|l|}{ ACESSO AO CEF } \\
\hline Amigo & $55 \%$ & $83 \%$ & $50 \%$ & $50 \%$ & $83 \%$ & $20 \%$ \\
\hline Paciente & - & - & $21 \%$ & - & - & - \\
\hline Médico & $5 \%$ & $17 \%$ & - & - & $17 \%$ & $20 \%$ \\
\hline Outro & $40 \%$ & - & $29 \%$ & $50 \%$ & - & $60 \%$ \\
\hline
\end{tabular}

Fonte: Elaborado pelos autores.

Os aspectos clínicos dos atendimentos realizados na Clínica Escola de Fisioterapia são detalhados na tabela 3: 
Tabela 3. Aspectos Clínicos dos atendimentos realizados na CEF do IESC-FAG.

\begin{tabular}{lcccccc}
\hline VARIÁVEIS & Orto & Neuro & Dermato & Cardio & Geri & Urogi \\
\hline $\begin{array}{l}\text { PRIMEIRO } \\
\text { ATENDIMENTO } \\
\text { FISIOTERAPÊUTICO }\end{array}$ & & & & & & \\
\hline Sim & $55 \%$ & $8 \%$ & $57 \%$ & $100 \%$ & $100 \%$ & $70 \%$ \\
\hline Não & $45 \%$ & $92 \%$ & $43 \%$ & - & - & $30 \%$ \\
\hline $\begin{array}{l}\text { PRIMEIRA VEZ NA } \\
\text { CEF }\end{array}$ & & & & & & \\
\hline Sim & $75 \%$ & $42 \%$ & $57 \%$ & $100 \%$ & $95 \%$ & $40 \%$ \\
\hline Não & $25 \%$ & $58 \%$ & $43 \%$ & - & $5 \%$ & $60 \%$ \\
\hline $\begin{array}{l}\text { CONHECE O } \\
\text { DIAGNÓSTICO CLÍNICO }\end{array}$ & $95 \%$ & $100 \%$ & $100 \%$ & $100 \%$ & $100 \%$ & $100 \%$ \\
\hline Sim & $5 \%$ & - & - & - & - & - \\
\hline Não & & & & & & \\
\hline $\begin{array}{l}\text { REALIZA FISIOTERAPIA } \\
\text { PARTICULAR }\end{array}$ & $5 \%$ & - & - & - & - & $5 \%$ \\
\hline Sim & $95 \%$ & $100 \%$ & $100 \%$ & $100 \%$ & $100 \%$ & $95 \%$ \\
\hline Não & & & & & & \\
\hline
\end{tabular}

Fonte: Elaborado pelos autores.

Os aspectos interpessoais na interação usuário e estagiário, são descritos na tabela 4:

Tabela 4. Aspectos interpessoais na interação usuário e estagiário da CEF do IESC-FAG.

\begin{tabular}{lcccccc}
\hline VARIÁVEIS & Orto & Neuro & Dermato & Cardio & Geri & Urogi \\
\hline $\begin{array}{l}\text { ESCLARECIMENTO DE } \\
\text { DÚVIDAS }\end{array}$ & & & & & & \\
\hline Ruim & - & $8 \%$ & - & - & $8 \%$ & - \\
\hline Bom & $25 \%$ & $17 \%$ & $21 \%$ & $25 \%$ & - & $20 \%$ \\
\hline Ótimo & $45 \%$ & $33 \%$ & $43 \%$ & $50 \%$ & $92 \%$ & $20 \%$ \\
\hline Excelente & $30 \%$ & $42 \%$ & $36 \%$ & $25 \%$ & - & $60 \%$ \\
\hline $\begin{array}{l}\text { SEGURANÇA DO } \\
\text { ESTAGIÁRIO }\end{array}$ & & & & & & \\
\hline Ruim & - & - & - & - & - & - \\
\hline Bom & $45 \%$ & $17 \%$ & $21 \%$ & $25 \%$ & - & $40 \%$ \\
\hline Ótimo & $30 \%$ & $75 \%$ & $36 \%$ & $50 \%$ & $92 \%$ & $10 \%$ \\
\hline Excelente & $25 \%$ & $8 \%$ & $43 \%$ & $25 \%$ & $8 \%$ & $50 \%$ \\
\hline $\begin{array}{l}\text { AVALIAÇÃO INTEGRAL } \\
\text { PELO ESTAGIÁRIO }\end{array}$ & & & & & & \\
\hline Ruim & $35 \%$ & $17 \%$ & - & - & - & $40 \%$ \\
\hline Bom & $30 \%$ & $83 \%$ & $7 \%$ & $25 \%$ & - & $20 \%$ \\
\hline Ótimo & $30 \%$ & - & $57 \%$ & $75 \%$ & $100 \%$ & $40 \%$ \\
\hline Excelente & $5 \%$ & - & $36 \%$ & - & - & - \\
\hline GENTILEZA DO ESTAGIÁRIO & & & & & & \\
\hline Ruim & - & - & - & - & - & - \\
\hline Bom & $30 \%$ & $9 \%$ & $14 \%$ & $25 \%$ & - & $10 \%$ \\
\hline Ótimo & $20 \%$ & $83 \%$ & $36 \%$ & $25 \%$ & $92 \%$ & $30 \%$ \\
\hline Excelente & $50 \%$ & $8 \%$ & $50 \%$ & $50 \%$ & $8 \%$ & $60 \%$ \\
\hline ABORDAGEM DO ESTAGIÁRIO & & & & & & \\
\hline
\end{tabular}




\begin{tabular}{lcccccc}
\hline Bom & $20 \%$ & $8 \%$ & - & - & - & $10 \%$ \\
\hline Ótimo & $25 \%$ & $92 \%$ & $64 \%$ & $75 \%$ & $100 \%$ & $30 \%$ \\
\hline Excelente & $55 \%$ & - & $36 \%$ & $25 \%$ & - & $60 \%$ \\
\hline GARANTIA DE PRIVACIDADE & & & & & & \\
\hline Ruim & - & - & - & - & - & - \\
\hline Bom & $45 \%$ & $7 \%$ & $7 \%$ & - & - & $40 \%$ \\
\hline Ótimo & $15 \%$ & $50 \%$ & $50 \%$ & $25 \%$ & $100 \%$ & $30 \%$ \\
\hline Excelente & $40 \%$ & $43 \%$ & $43 \%$ & $75 \%$ & - & $30 \%$ \\
\hline
\end{tabular}

Fonte: Elaborado pelos autores.

$\mathrm{Na}$ tabela 5, são demonstrados o acesso ao serviço e atendimento da recepção e pessoal de apoio da CEF do IESC FAG:

Tabela 5. Acesso ao serviço e atendimento da recepção e pessoal de apoio da CEF do IESC-FAG.

\begin{tabular}{|c|c|c|c|c|c|c|}
\hline VARIÁVEIS & Orto & Neuro & Dermato & Cardio & Geri & Urogi \\
\hline \multicolumn{7}{|c|}{$\begin{array}{l}\text { FACILIDADE NO } \\
\text { AGENDAMENTO DA } 1^{\text {a }} \\
\text { CONSULTA }\end{array}$} \\
\hline Ruim & - & $8 \%$ & - & - & - & - \\
\hline Bom & $30 \%$ & $50 \%$ & $14 \%$ & - & - & $20 \%$ \\
\hline Ótimo & $30 \%$ & $42 \%$ & $14 \%$ & $50 \%$ & $100 \%$ & $50 \%$ \\
\hline Excelente & $40 \%$ & - & $72 \%$ & $50 \%$ & - & $30 \%$ \\
\hline \multicolumn{7}{|c|}{$\begin{array}{l}\text { FACILIDADE NO } \\
\text { GENDAMENTOS DAS SESSÕES } \\
\text { FISIOTERAPÊUTICA }\end{array}$} \\
\hline Ruim & - & - & - & - & - & - \\
\hline Bom & $35 \%$ & $50 \%$ & $7 \%$ & - & - & $10 \%$ \\
\hline Ótimo & $25 \%$ & $50 \%$ & $39 \%$ & $50 \%$ & $100 \%$ & $70 \%$ \\
\hline Excelente & $40 \%$ & - & $64 \%$ & $50 \%$ & - & $20 \%$ \\
\hline \multicolumn{7}{|c|}{$\begin{array}{l}\text { TEMPO DE ESPERA } \\
\text { POR ATENDIMENTO }\end{array}$} \\
\hline Ruim & - & - & - & - & - & - \\
\hline Bom & $35 \%$ & $58 \%$ & $21 \%$ & - & - & $40 \%$ \\
\hline Ótimo & $40 \%$ & $42 \%$ & $43 \%$ & $25 \%$ & $100 \%$ & $30 \%$ \\
\hline Excelente & $25 \%$ & - & $36 \%$ & $75 \%$ & - & $30 \%$ \\
\hline \multicolumn{7}{|c|}{$\begin{array}{l}\text { GENTILEZA E DISPONIBILIDADE } \\
\text { NA RECEPÇÃO }\end{array}$} \\
\hline Ruim & - & $8 \%$ & - & - & - & - \\
\hline Bom & $35 \%$ & $8 \%$ & $22 \%$ & - & - & $30 \%$ \\
\hline Ótimo & $40 \%$ & $42 \%$ & $21 \%$ & $50 \%$ & $100 \%$ & $30 \%$ \\
\hline Excelente & $25 \%$ & $42 \%$ & $57 \%$ & $50 \%$ & - & $40 \%$ \\
\hline
\end{tabular}

Fonte: Elaborado pelos autores.

Figura 2: Recepção do CEPS do IESC-FAG. 


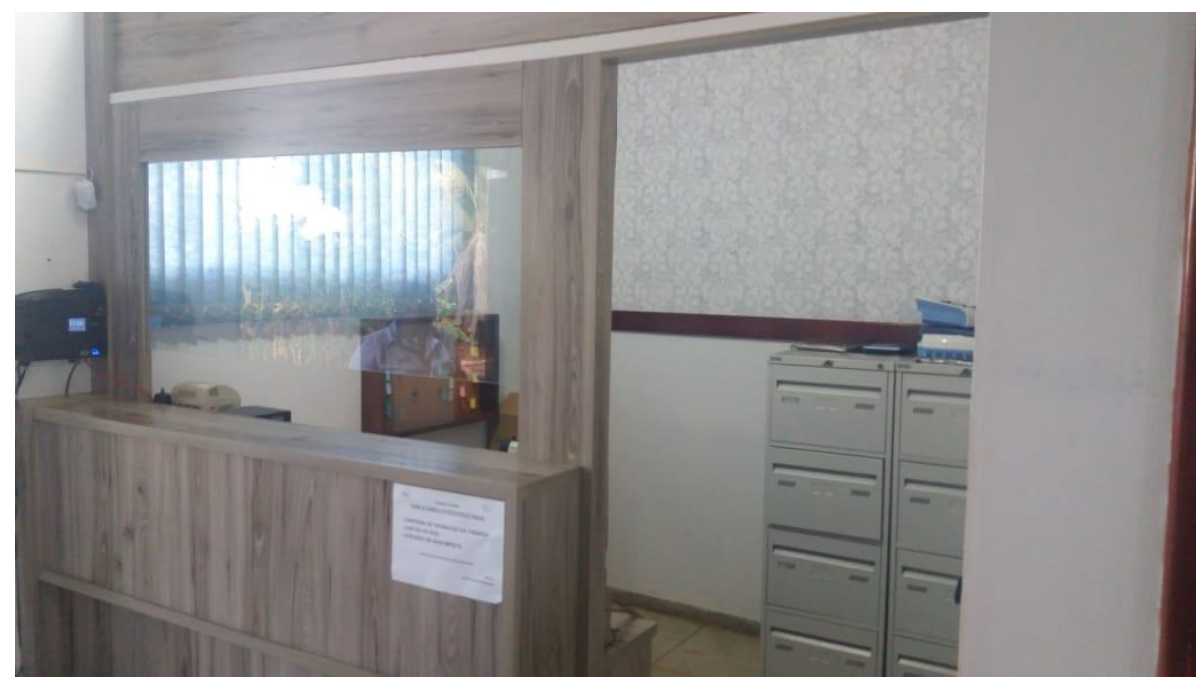

Fonte: Arquivo pessoal dos autores.

A estrutura da CEF também foi avaliada pelos pacientes quanto aos seguintes critérios: conforto na sala de espera, conforto na sala de atendimento, facilidade de locomoção nas instalações e condições gerais de higiene dos ambientes, com seus respectivos percentuais apresentados na tabela 6 , a seguir.

Tabela 6. Ambiente físico da CEF do IESC-FAG.

\begin{tabular}{|c|c|c|c|c|c|c|}
\hline VARIÁVEIS & Orto & Neuro & Dermato & Cardio & Geri & Urogi \\
\hline \multicolumn{7}{|c|}{$\begin{array}{l}\text { CONFORTO NA SALA } \\
\text { DE ESPERA }\end{array}$} \\
\hline Péssimo & - & - & - & - & - & $10 \%$ \\
\hline Ruim & - & $8 \%$ & - & - & - & - \\
\hline Bom & $40 \%$ & $87 \%$ & $43 \%$ & - & $8 \%$ & $60 \%$ \\
\hline Ótimo & $35 \%$ & $25 \%$ & $36 \%$ & $50 \%$ & $92 \%$ & $20 \%$ \\
\hline Excelente & $25 \%$ & - & $21 \%$ & $50 \%$ & - & $10 \%$ \\
\hline \multicolumn{7}{|c|}{$\begin{array}{l}\text { CONFORTO NO AMBIENTE } \\
\text { DE ATENDIMENTO }\end{array}$} \\
\hline Ruim & - & $8 \%$ & - & - & - & - \\
\hline Bom & $30 \%$ & $59 \%$ & $14 \%$ & - & - & $40 \%$ \\
\hline Ótimo & $45 \%$ & $33 \%$ & $57 \%$ & $50 \%$ & $100 \%$ & $50 \%$ \\
\hline Excelente & $25 \%$ & - & $29 \%$ & $50 \%$ & - & $10 \%$ \\
\hline \multicolumn{7}{|c|}{$\begin{array}{l}\text { FACILIDADE DE LOCOMOÇÃO } \\
\text { NAS INSTALAÇÕES }\end{array}$} \\
\hline Ruim & - & - & - & - & - & - \\
\hline Bom & $35 \%$ & $67 \%$ & $36 \%$ & - & - & $70 \%$ \\
\hline Ótimo & $30 \%$ & $33 \%$ & $43 \%$ & $25 \%$ & $100 \%$ & $20 \%$ \\
\hline Excelente & $35 \%$ & - & $21 \%$ & $75 \%$ & - & $10 \%$ \\
\hline \multicolumn{7}{|c|}{$\begin{array}{l}\text { CONDIÇÕES GERAIS } \\
\text { DE HIGIENE DOS AMBIENTES }\end{array}$} \\
\hline Ruim & - & - & - & - & - & - \\
\hline Bom & $30 \%$ & $17 \%$ & $21 \%$ & - & - & $50 \%$ \\
\hline Ótimo & $40 \%$ & $83 \%$ & $36 \%$ & $25 \%$ & $100 \%$ & $20 \%$ \\
\hline Excelente & $30 \%$ & - & $43 \%$ & $75 \%$ & - & $30 \%$ \\
\hline
\end{tabular}

Fonte: Elaborado pelos autores. 
Figura 3: Sala de Espera da CEF do IESC-FAG - Entrada para a sala de Fisioterapia.

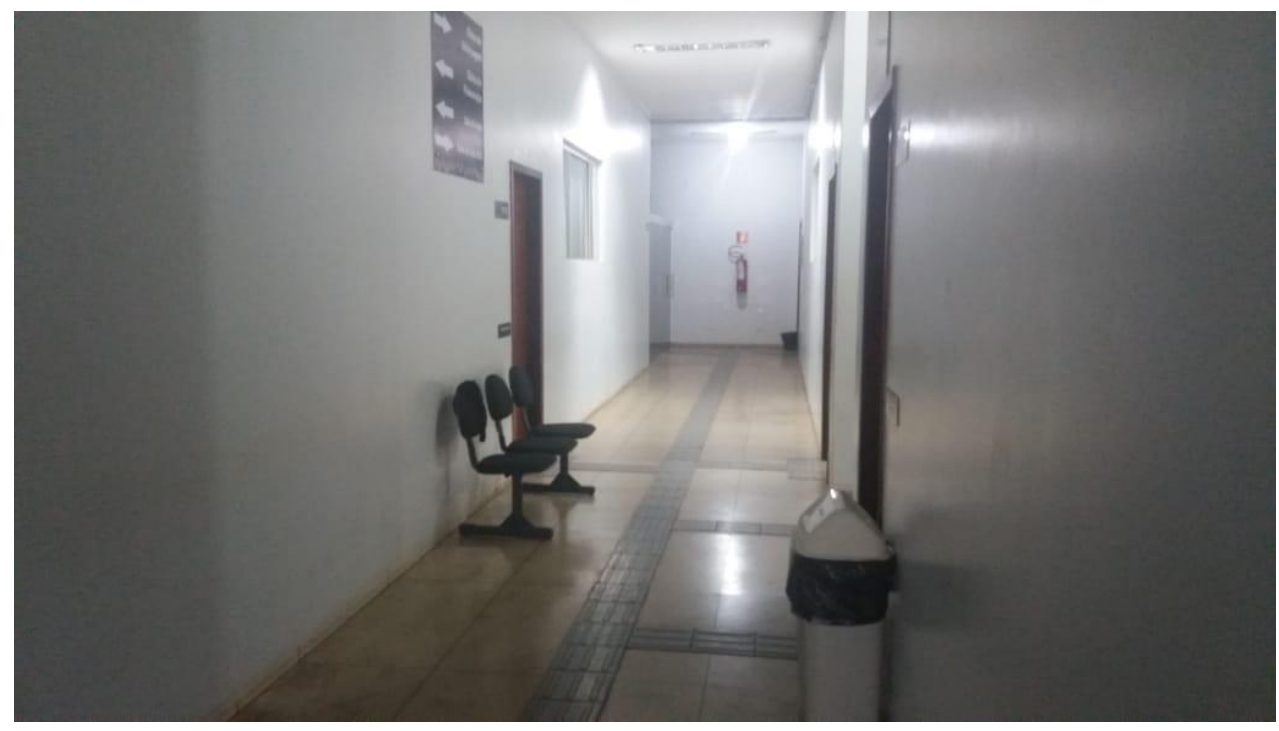

Fonte: Arquivo pessoal dos autores.

Figura 4: Sala de Atendimento de Fisioterapia da CEF do IESC-FAG.

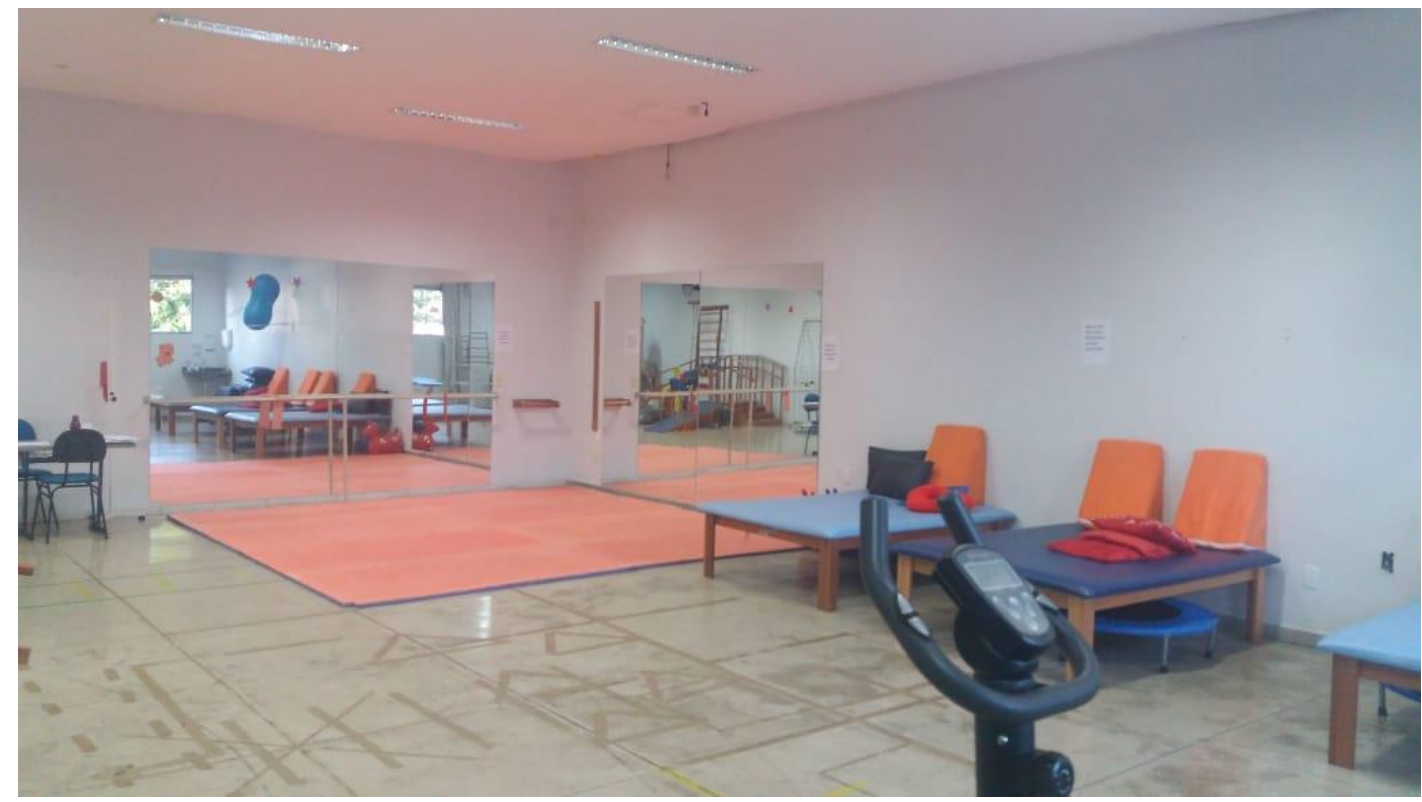

Fonte: Arquivo pessoal dos autores. 
Figura 5: Sala de Atendimento (Sala de eletroterapia) de Fisioterapia da CEF do IESCFAG.

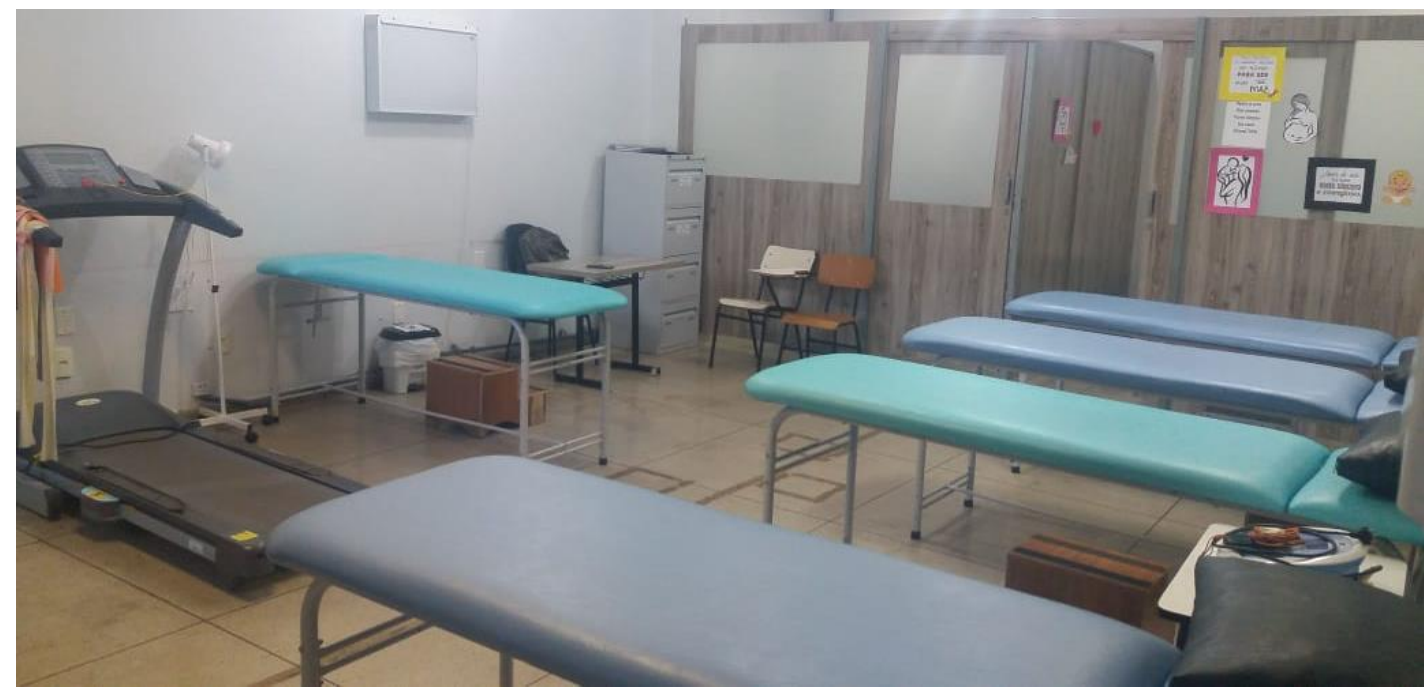

Fonte: Arquivo pessoal dos autores.

\section{DISCUSSÃO}

Os dados sociodemográficos e clínicos do presente estudo corroboram com outros estudos (SUDA et al. 2009; BÁLICA et al. 2016), que apresentou o maior índice de representatividade pelo gênero feminino, baixo nível de escolaridade e renda familiar entre 1 e 3 salários mínimos. O presente trabalho evidenciou que na área de atendimento de dermato - funcional, o nível de renda familiar é entre 4 e 6 salários mínimos e grau de escolaridade também é mais elevado.

No entanto, o índice "acesso a clínica escola" teve uma maior porcentagem de conhecimentos da CEF por 'AMIGOS', relacionando assim ao conhecimento da população referente aos atendimentos prestado na clínica escola corroborando assim com o estudo de Cattani (2016).

$\mathrm{Na}$ amplitude de atendimento pelos estagiários de Fisioterapia na CEF, descritos na tabela 2, foi questionado ao entrevistado, se "era a primeira vez em que havia sido atendido por profissionais da área". Um elevado índice relatou que "SIM", que seria a primeira vez, porém $92 \%$ dos pacientes de neurofuncional, afirmaram já terem sido atendidos anteriormente. Essa diversidade em relação à área de neurofuncional se diz respeito ao fato de as sequelas de doença neurológica serem mais incapacitantes, com isso a preocupação do indivíduo de procurar de maneira mais rápida o tratamento fisioterapêutico. 
Todos os participantes desse estudo afirmaram estar cientes que os atendimentos oferecidos na CEF eram realizados por acadêmicos estagiários, sob supervisão de professores fisioterapeutas, e que a dinâmica de atendimento seria em forma de "rodízio", em que o paciente seria atendido por diferentes estagiários conforme suas necessidades. Diante desse modelo de atendimento, todos os participantes o consideraram como satisfatório.

Diferentes estudos mostram que os atendimentos oferecidos por instituições de ensino superior da área da saúde tem sido cada vez mais procurados pela comunidade. $E$ estas, por sua vez, desenvolvem um importante papel na formação dos acadêmicos e contribuem para a qualidade de vida, promoção da saúde e prevenção de agravos, tratamento e reabilitação adequada. Essa procura pode estar relacionada a diminuição do preconceito e estigma relacionado ao atendimento feito por um profissional ainda em formação (SUDA et al. 2009; MACHADO \& NOGUEIRA, 2008; SILVA et al. 2014).

Observou-se na variável "se era a primeira vez em que o paciente estava sendo atendido na Clínica Escola de Fisioterapia", que grande parte dos participantes estavam pela primeira vez sendo atendido na CEF, porem $60 \%$ da área de uroginecologia, já havia sido atendido por profissionais de fisioterapia na CEF anteriormente, em outras áreas de atendimento. Isso acontece porque o indivíduo pode ter patologias e/ou sinais e sintomas diversos, que se enquadrem e outras áreas da fisioterapia, corroborando com o estudo de Gervaz et al. (2017).

Em relação ao diagnóstico clínico, foi evidenciado um alto índice de pacientes que conhece seu próprio diagnóstico e não faz uso de fisioterapia em clínica particular assim como no estudo de Barbosa (2014).

A qualidade na assistência à saúde inclui a empatia do cuidado com o cliente, interagindo melhor com o indivíduo a fim de identificar o que lhe agrada. A satisfação dos clientes vem sendo cada vez mais analisada para o crescimento dos serviços em saúde pública (SANTOS et al. 2012).

Identificar a satisfação dos pacientes quanto aos serviços recebidos na área da saúde, tem sido cada vez mais utilizado como um importante parâmetro definidor para avaliação da qualidade dos atendimentos e serviços prestados na área da saúde (GABE et al. 2018).

Referente à dimensão de receptividade por todos os profissionais no ambiente de recepção e atendimento da CEF, a segurança transmitida pelo estagiário, gentileza, 
respeito e explicações dadas pelo estagiário, no presente estudo teve um alto índice obtido de relatos que ditam ser "ÓTIMO", correlacionando com outras pesquisas (BÁLICA et al. 2016; BRITO, JESUS, FERNANDES, 2012).

Os dados obtidos nesse estudo demonstram como é importante o profissional fisioterapeuta estabelecer uma adequada comunicação com seu paciente escutando - 0 , esclarecendo suas dúvidas, proporcionando confiança e segurança. Essa relação interpessoal entre profissional e paciente é muito importante e valorizada pelo usuário dos serviços de saúde, confirmando assim o os dados do estudo de Barreto (2013).

Em relação aos itens "esclarecimento de dúvidas" e "avaliação integral pelo estagiário", observou-se percentuais significativos da opção "RUIM", sendo no atendimento Neurofuncional (8\%), Geriatria (8\%) quanto ao esclarecimento de dúvidas; Ortopedia (35\%), Neurofuncional (17\%) e Uroginecologia (40\%) quanto a avaliação integral realizada pelo estagiário. Essas negativas podem ser justificadas pela insegurança dos estagiários de responderem aos questionamentos dos pacientes, ou pela falta de investigação clínica das patologias, visto que se percebe ênfase apenas aos sintomas clínicos dos pacientes.

A comunicação e abordagem do estagiário ao paciente precisam ser respaldadas e executadas com cautela, pois situações como: uma ideia mal expressada; demonstração de impaciência ou intolerância; desatenção na anamnese e exame físico; falta de empatia; preconceito; constrangimento, entre outras, podem comprometer todo o processo terapêutico, ou estimular o abandono por parte do paciente e piora do seu estado de saúde (SANTOS, GARCIA, 2009).

Quanto ao acesso à CEF destaca-se que apenas na especialidade de Neurofuncional há insatisfação quanto a facilidade do agendamento da primeira consulta, em contrapartida, para a especialidade Geriatria foi atribuída por unanimidade o conceito de ótimo à essa variável. Considerando a importância da continuidade do tratamento fisioterapêutico, o agendamento das sessões de fisioterapia após a primeira consulta é fundamental, e nessa variável as especialidades de cardiologia e geriatria foram as que evidenciaram maior satisfação pelos pacientes.

O tempo de espera por atendimento na CEF foi considerado adequado, visto que nenhum dos participantes da pesquisa atribuíram o critério "RUIM". As especialidades de Cardiologia e Geriatria foram as que apresentaram os maiores percentuais de satisfação do usuário. 
Diante dos dados referentes ao processo de agendamento de consultas e disponibilidade da recepção, percebe-se a necessidade de revisão sobre o processo de acolhimento dos pacientes, visto que ele é também definido como ideal para classificação de risco, e coordenador para os encaminhamentos necessários a cada paciente. Para isso, a escuta qualificada se torna uma ferramenta indispensável (SCHOLZE, 2014).

Quanto ao tempo de espera por atendimento, os dados mostram que as especialidades de Cardiologia e Geriatria são consideradas as mais ágeis, porém, todas as especialidades foram consideradas satisfatórias nesse quesito.

Os dados evidenciam um pequeno percentual (10\%) de insatisfação quanto ao conforto da sala de espera por parte dos pacientes da especialidade de Uroginecologia. Quanto ao conforto do ambiente de atendimento, os pacientes de Neurofuncional também demonstraram insatisfação (8\%). A facilidade de locomoção nas instalações da CEF e as condições de higiene foram consideradas satisfatórias pelos pacientes.

O ambiente em que são oferecidos serviços de saúde pode ser definido como aquele que apresenta organização sistemática, que devem facilitar a prática profissional, contribuindo para alcance da qualidade na assistência prestada, podendo interferir direta ou indiretamente (ANDRADE, 2016).

Esse índice negativo sobre o conforto no ambiente de atendimento pode ser justificado pelo fato de não ter atendimento privativo dentro da CEF, pois dentro da sala onde são realizados os atendimentos da área de Neurofuncional, o espaço é aberto e amplo, sem ter muita privacidade no atendimento, e alguns pacientes relataram sentir vergonha, ou constrangimento por estarem sendo vistos por todos.

Constatou-se que na variável "facilidade de locomoção nas instalações" e "condições de higiene", os voluntários relatam índice "ÓTIMO", correlacionando assim este estudo com Guzzo e Fassiolo (2011), onde descreve que é importante ter facilidade para transitar nos ambientes de atendimento, assim como a garantia de higiene adequada.

Quando questionados sobre a possibilidade de retornar a CEF para continuidade do tratamento ou conforme necessidade do paciente, todos responderam "SIM", sendo esse, mais um indicador de satisfação do paciente pelo atendimento recebido. Além disso, questionou-se se eles indicariam a CEF para outras pessoas, e novamente, por unanimidade todos afirmaram que recomendariam, correlacionando com o estudo de Viana et al. (2014). 
A gentileza e disponibilidade da recepção apresentou avaliação considera "RUIM" apenas na especialidade de Neurofuncional. Sendo Cardiologia e Geriatria, mantiveram os percentuais mais elevados.

No âmbito dos serviços de saúde, o acolhimento não se restringe apenas a recepção do paciente, mas sim uma diretriz operacional, em que o acesso e o acolhimento se articulam e se complementar para alcance de práticas integrais de cuidado e serviços de saúde eficientes e resolutivos (BRASIL, 2010).

\section{CONSIDERAÇÕES FINAIS}

Foi apontada nesta pesquisa a importância de se avaliar a satisfação dos usuários em geral, e os serviços prestados na CEF. A maioria dos participantes demonstraram estar satisfeitos com os aspectos pesquisados, com apenas algumas insatisfações, em que é preciso dispor de ambientes mais privativos, sala de espera para que melhore o conforto dos pacientes e estratégias que agilizam o tempo de espera. Percebeu-se também a necessidade de aprimoramento do acolhimento dos pacientes, desde a recepção, passando pelo atendimento realizado e pelo agendamento de retorno.

Desta forma, todas as informações colhidas nesse estudo são de suma importância para compreender a percepção dos usuários e suas reais necessidades, e a partir disso fomentar o aprimoramento contínuo dos serviços oferecidos. Assim, contribuir significativamente para formação dos futuros fisioterapeutas, elevando o nível de qualidade dos serviços oferecidos pela CEF do IESC FAG.

\section{REFERÉNCIAS}

ANDRADE, Carina Manuela Mouquinho. Ambiente da Prática Profissional de Enfermagem - Impacto na Qualidade dos Cuidados de Saúde. Dissertação de Mestrado em Enfermagem. Escola Superior de Enfermagem de Lisboa, 2019. Disponível em: http://hdl.handle.net/10400.26/17248 Acessado em: 01/07/2020

BÁLICA, Solange Cruz Ponte; MAGAZONI Valéria Sachi; BRAGA, Thaís Ferreira; GUIMARÃES, Élcio. Avaliação da satisfação dos pacientes em uma clínica de saúde de fisioterapia em Caxias-MA. REAS, Revista Eletrônica Acervo Saúde, 2016. Vol. 8 (2), 861-667. Disponível em: https://www.acervosaude.com.br/doc/8 2016.pdf Acessado em: $01 / 07 / 2020$

\section{BARBOSA, Elcio Cardoso. Satisfação da assistência fisioterapêutica: uma} avaliação de usuário no município de Esperança-PB. 2014. Trabalho de Conclusão de Curso. Departamento de Fisioterapia do Centro de Ciências Biológicas e da Saúde 
da Universidade Estadual da Paraíba. Disponível em:

https://pdfs.semanticscholar.org/f1c8/6c49f505c510fea7112144165ad80198f827.pdf

Acessado em: 01/07/2020

BARRETO, Marcos Túlio. Qualidade no atendimento de uma unidade de serviço do SUS: aplicação do modelo SERVQUAL para mensurar a qualidade dos serviços prestados na clínica escola de fisioterapia da UEPB. Trabalho de conclusão do curso de Fisioterapia. Universidade Estadual da Paraíba, Campina Grande: 2013. Disponível: http://dspace.bc.uepb.edu.br/jspui/bitstream/123456789/5046/1/PDF\%20\%20Mona\%C3\%ADsa\%20Targino\%20Barreto.pdf Acessado: 01/07/2020

BRASIL. Resolução CNE/CES 4/2002. Diretrizes Curriculares Nacionais. Diário Oficial da União, Brasília, 4 de março de 2002. Seção 1, p. 11.

BRASIL. Ministério da Saúde. Acolhimento nas Práticas de Produção de Saúde. Brasília (Brasil): Ministério da Saúde, 2010. Disponível em:

https://bvsms.saude.gov.br/bvs/publicacoes/acolhimento praticas producao saude.pdf Acessado em: 01/07/2020

BRITO, Tiago Almeida; JESUS, Cassio Soares; FERNANDES, Murilo Henrique. Fatores associados à satisfação dos usuários em serviços de fisioterapia. Revista Baiana de Saúde Pública, v. 36, n. 2, p. 514, 2012. Disponível em: http://files.bvs.br/upload/S/01000233/2012/v36n2/a3247.pdf Acessado em: 01/07/2020

CATTANI, Ingrid Costa; BORRILE, Rodrigo Lima; TRAVERSO, Mateus Eduardo Duarte. Avaliação Da Satisfação Dos Usuários De Fisioterapia Em Uma Clínica-Escola De Uma Universidade Do Meio-Oeste Catarinense. Fisioterapia em Ação. Anais Eletrônicos, 2016. Disponível em: https://portalperiodicos.unoesc.edu.br/fisioterapiaemacao/article/view/10217/5380 Acessado em: 01/07/2020

GABE, Milena Bancer; ROSA, Áurea Maria Soares da; GARCIA, Leda Soares Brandão; AMBROSIO, Pedro Gabriel; MADEIRA, Kristhian. Avaliação do grau de satisfação de pacientes atendidos no serviço de medicina de uma clínica escola. Arq.; Catarin Med. 2018; jul.set. 47(3):146-158. Disponível em:

http://www.acm.org.br/acm/seer/index.php/arquivos/article/view/462/279 Acessado em: 01/07/2020

GAIAD, Thiago; PEIXOTO, Silvia; GONÇALVES, Daniel Mendes. Análise da eficácia do estágio supervisionado em fisioterapia na formação profissional: Uma visão do egresso. Arq. Ciênc. Saúde Unipar, Umuarama, 9(2), mai./ago. p.65-70. Disponível em: https://revistas.unipar.br/index.php/saude/article/viewFile/200/174 Acessado em: 30/06/2020

GERVAZ, Valriele Rodrigues Silva; FERREIRA, Ana Paula Santos; SILVA, Ariella Rosseti; AZEVEDO, Naara Caroline; MORAES, Yde Amendola. Avaliação Da Satisfação Dos Pacientes Atendidos Em Uma Clínica-Escola De Fisioterapia De Registro, SP. 17 Congresso Nacional de Iniciação Científica. Centro Universitário Ítalo Brasileiro, 2017. Disponível em: http://conicsemesp.org.br/anais/files/2017/trabalho-1000024368.pdf Acessado em: 01/07/2020 
GUZZO, Simone; FASSIOLLO, Carlos Eduardo. Avaliação da satisfação dos usuários de serviços de fisioterapia na clínica escola de pesquisa e atendimento em fisioterapia (CEPAF) da Universidade do Oeste de Santa Catarina (Unoesc). Revista Digital, Buenos Aires, ano 16, n. 163, dez. 2011. Disponível em:

http://www.efdeportes.com/efd163/avaliacao-da-satisfacao-dos-servicos-de-

fisioterapia.htm Acessado em: 01/07/2020

JESUS, André; DUARTE Judite Maria Machado; FIALHO, Cristiane Gonçalves de Oliveira; LIMONGE, Célber; SETTE, Fabricío. Avaliação da satisfação dos pacientes atendidos na Clínica Escola do departamento de Fisioterapia da Univiçosa. Anais II SIMPAC - Volume 2 - n.1 - Viçosa-MG - jan. - dez. - 2010 - p. 123-128. Disponível em: https://academico.univicosa.com.br/revista/index.php/RevistaSimpac/article/view/266 Acessado em: 01/07/2020

MACHADO, NP; NOGUEIRA, LT. Avaliação da Satisfação dos usuários de serviços de fisioterapia, Rev.Bras Fisioter, São Carlos, volume 12, número 5, 401 - 8, set/out. 2008. Disponível em: https://www.scielo.br/pdf/rbfis/v12n5/a10v12n5.pdf Acessado em: 01/07/2020

SANTOS, Jaqueline Bontenpo; BICALHO, Karina Andrade; BORGES, Maria Beatriz e Silva. Características e níveis de satisfação dos pacientes atendidos na clinica- escola de fisioterapia da Universidade Católica de Brasília. EFDeportes.com, Revista Digital. Buenos Aires, Año 17, № 171, Agosto de 2012. Disponível em: https://www.efdeportes.com/efd171/satisfacao-dos-pacientes-na-clinica-de-fisioterapia.htm Acessado em: 01/07/2020

SANTOS, Rafael de Lacerda; GARCIA, Rosamaria Rodrigues. Avaliação da Qualidade Do Atendimento Prestado Na Clínica De Fisioterapia Da Universidade Municipal De São Caetano Do Sul. Revista Brasileira de Ciências da Saúde, ano VII, no 20, abr/jun 2009. Disponível em:

https://seer.uscs.edu.br/index.php/revista ciencias saude/article/view/316/141 Acessado em: 01/07/2020

SCHOLZE, Alessandro da Silva. Acolhimento com classificação de risco para a Estratégia Saúde da Família: a prática em uma unidade docente-assistencial. Rev Bras Med Fam Comunidade. 2014;9(31):219-26. Disponível em: https://rbmfc.org.br/rbmfc/article/view/637 Acessado em: 01/07/2020

SILVA, Karla Camila Correia da; NUNES, Rodrigo Disconzi; ARGEO, Rafael Fernando; TAKADA; Jacqueline Aparecida Philipino; OLIVEIRA, Leonardo Lopes; SILVA, Mariana Pereira. Nível de satisfação de atendimento fisioterapêutico realizados na clinica escola de fisioterapia do Centro Universitario UNIRG no Municipio de Gurupi-TO. Revista Amazonia: Science \& Health 2 (4), 24-28, 2014. Disponível em: http://ojs.unirg.edu.br/index.php/2/article/view/555 Acessado em: 01/07/2020

SUDA, Eneida Yuri; UEMURA, Missae Dora; VELASCO, Eliane. Avaliação da satisfação dos pacientes atendidos em uma clínica-escola de Fisioterapia de Santo André, SP.

Fisioterapia e Pesquisa, São Paulo, v.16, n.2, p.126-31, abr./jun. 2009 Disponível em: https://www.scielo.br/pdf/fp/v16n2/06.pdf Acessado em: 01/07/2020 
VIANA, jenifer Rossiele Silva; RAIOCOVITCH, Tayara; GOLIAS, Andrey Rogério; PETERNELLA, Fabiana Magalhães Navarro; MENDES, Fernando Cordeiro Vila; GRABOWSKI, Jorge. Avaliação Da Satisfação Com A Fisioterapia De Pacientes Atendidos Em Uma Clínica Escola De Maringá - Pr, Revista Uningá. Volume.17, numero. 2, pag. $16-21$, jan/mar. 2014. Disponível em:

http://revista.uninga.br/index.php/uningareviews/article/view/1493 Acesso em: 01/07/2020 